Research Article

\title{
An Improved Adaptive Passive Coordinated Control for Generator Excitation and STATCOM
}

\author{
Chaofan Du $\mathbb{D}^{1}{ }^{1}$ Lei Zhang $\mathbb{D D}^{1}{ }^{1}$ Yuanhui Tang, ${ }^{2}$ Jiahao Zhu, ${ }^{1}$ and Kaifeng Wang ${ }^{1}$ \\ ${ }^{1}$ School of Electronics and Information, Xian Polytechnic University, Xian, Shaanxi 710048, China \\ ${ }^{2}$ Shaanxi Provincial Institute of Metrology, Xian, Shaanxi 710052, China \\ Correspondence should be addressed to Lei Zhang; carol1208@163.com
}

Received 10 December 2020; Revised 23 June 2021; Accepted 20 July 2021; Published 3 August 2021

Academic Editor: Ciro Núñez-Gutiérrez

Copyright (c) 2021 Chaofan Du et al. This is an open access article distributed under the Creative Commons Attribution License, which permits unrestricted use, distribution, and reproduction in any medium, provided the original work is properly cited.

An improved adaptive backstepping passive coordinated control strategy is proposed to deal with the uncertainty of damping coefficient and mechanical power and external disturbance on the system stability. A nonlinear coordinated controller of generator excitation and STATCOM (static synchronous compensator) is designed to improve its adaptability. The adaptive estimation laws of damping coefficient and mechanical power are designed by using I\&I (immersion and invariance) adaptive control to improve the adaptive ability of the controller. Based on the backstepping recursive method, the control law of STATCOM is designed and combines the passivity theory, thus acquiring the control law of generator excitation. In the backstepping design, the derivative of the virtual control input and external disturbance are considered to be uncertain, and the nonlinear damping algorithm is introduced to reduce the "calculation explosion." Simulation results show that the designed controller improves the stability of the power system and shows strong adaptability and robustness.

\section{Introduction}

At present, the scale and structure of power grid is more and more large and complex, and the transmission system has developed into a large capacity, long-distance, and ultrahigh voltage transmission form $[1,2]$. The following problem is the stability of the power system. Flexible AC Transmission System (FACTS) can solve the stability problem of the power system economically and effectively $[3,4]$. The static synchronous compensator (STATCOM) is a good FACTS device to improve the voltage quality of the control installation point, adjust the reactive power of the power grid, increase the transmission capacity of the line, and improve the transient stability of the power system $[5,6]$. The generator excitation system has always played an important role in solving the stability problem of the power system [7]. In the transmission system equipped with STATCOM, the interaction of the different controllers is the kernel that impacts the system dynamics. Therefore, it is extremely valuable to research on how to design the coordinated controller of generator excitation and STATCOM.
Many uncertain parameters in the power system are the main factors affecting the stability of the transmission system [8]. When establishing the mathematical model of the transmission system equipped with STACOM, some approximate processing is usually done or the parameters are assumed to be known, leading to the existence of uncertain parameters [9]. However, when the system suffers from disturbances such as generator fault or fault load removal during operation, the current network structure of the system will be different from that of modeling. In the early stage, the linear control method [10] ignores the uncertainty in system modeling, and its design of parameters are not robust, when system changes. To solve the shortcomings of the linear control method, adopting nonlinear control methods to design the system has become a popular research subject in recent years, such as the target holographic feedback method, fuzzy control theory [11], sliding mode variable control method [12], passive control method [13], Hamiltonian theory [14], and adaptive backstepping method [15]. Among the above control methods, the adaptive backstepping method, as a simple and effective nonlinear 
control method, has been widely used in designing the coordinated controller of excitation and STACOM, achieving good results [15]. The designed controller based on the adaptive backstepping method [15] can only estimate a single parameter, and this algorithm has poor estimation ability for a single parameter. Inspired by [16], I\&I adaptive control algorithm can design parameter adaptive laws for multiple uncertain parameters in the system model at the same time, estimating multiple parameters and improving the estimation ability of parameters.

In addition, there is a problem of "calculation explosion" when using the backstepping method in higher-order systems. The existing literature mainly adopts dynamic surface control [17] and nonlinear tracking differentiator algorithm [18] to solve this problem. However, dynamic surface control can make the tracking error of the system converge exponentially to the bounded set, which is not globally asymptotically stable; the design process of nonlinear tracking differentiator algorithm is complex and difficult to understand. Pan et al. [19] regard the derivative of the backstepping virtual control as an uncertain term, introduce a robust term to solve the problem of "calculation explosion," and the system satisfies the global asymptotic stability. Therefore, this paper adopts the idea of [19] and introduces the nonlinear damping algorithm to deal with uncertain items. This algorithm has a simple design process, overcomes the defects of the above two control methods, and has strong robustness.

Contributions of this paper: considering the influence of the uncertain two parameters on the stability of generator excitation and STATCOM coordinated control system, based on I\&I adaptive control, the adaptive estimation laws are designed, which breaks through the certainty equivalence principle, solves the bottleneck problem of multiparameters' identification in the system, and enhances the adaptive ability of the controller. Moreover, the virtual control input and unknown disturbance in the system are regarded as uncertain items, and the nonlinear damping algorithm is used to compensate the uncertain items to solve the inverse problem.

\section{Passivity and Design Method}

Definition 1. Nonlinear systems as follows:

$$
\left\{\begin{array}{l}
\dot{x}=f(x)+g(x) u \\
y=h(x)
\end{array}\right.
$$

where $x \in R^{n}, u \in R^{m}$, and $f, g$, and $h$ are all fairly smooth function vectors.

If there is a semipositive definite function $V(x)$ ( $V$ : $x \in R, V(0)=0$ ), such that the following inequality,

$$
V(x(t))-V(x(0)) \leq \int_{0}^{t} y^{T}(\tau) u(\tau) \mathrm{d} \tau,
$$

holds for $\forall t \geq 0$ and $u \in R^{m}$, the nonlinear system (equation (1)) is called a passive system, where $V(x)$ is the energy storage function and equation (2) is called dissipation inequality.
Definition 2. For the passive system (1) and the energy storage function $V(x)$, there is a positive definite function $Q(x)$, such that

$$
V(x(t))-V(x(0)) \leq \int_{0}^{t} y^{T}(\tau) u(\tau) \mathrm{d} \tau-\int_{0}^{t} Q(x) \mathrm{d} \tau .
$$

If $\forall t \geq 0, u \in R^{m}$, and equation (3) holds, then the resultant system (equation (1)) is strictly passive.

According to equations (2) and (3), we can know that the zero output of the system is Lyapunov stability. If the system is strictly passive, then the zero output of the system is Lyapunov asymptotic stability [20].

Theorem 1. For a two-input system, an input-output pair $(u, y)$ is selected for which the relative degree is one order, and the system (equation (1)) can be converted into the following form:

$$
\left\{\begin{array}{l}
\dot{z}=q(z, y)+p(z, y) u_{2}, \\
\dot{y}=\alpha(z, y)+\beta_{1}(z, y) u_{1}+\beta_{2}(z, y) u_{2} .
\end{array}\right.
$$

The control law of (4) is

$$
\left\{\begin{array}{l}
u_{1}=\beta_{1}^{-1}(z, y)\left[-\beta_{2}(z, y) u_{2}(z)-\alpha(z, y)-\frac{\partial W}{\partial z} \widetilde{p}(z, y)+v\right], \\
u_{2}=\gamma(z),
\end{array}\right.
$$

where $\widetilde{p}(z, y)=q(z, 0)+p(z, 0) \gamma(z)$. Then, the Lyapunov function $W(z)$ such that

$$
\dot{W}=\frac{\partial W}{\partial z}(q(z, 0)+p(z, 0) \gamma(z))<-\alpha(\|z\|),
$$

where $\alpha$ is a $k$-class function.

Take the energy storage function $V(z)$ as

$$
V=W(z)+\frac{1}{2} y^{2}
$$

which satisfy $V \geq 0$ and $\dot{V} \leq 0$. Therefore, the system (equation (4)) can be guaranteed to be asymptotically stable.

It can be easily verified that the storage function satisfies for system (4), which proves the passivity of the pair [20].

\section{Power System Model}

The research object of this paper is the single machine infinite bus (SMIB) power system structure with STATCOM considering generator excitation control, as shown in Figure 1 . The generator excitation control equipment is connected to the power transmission line with the circuit breaker through the transformer, and STATCOM is installed at the midpoint of the line. Research shows that STATCOM can effectively improve the transmission capacity of the system, extend the transmission distance, and give full play to STATCOM's role in power system stability $[14,21]$. 


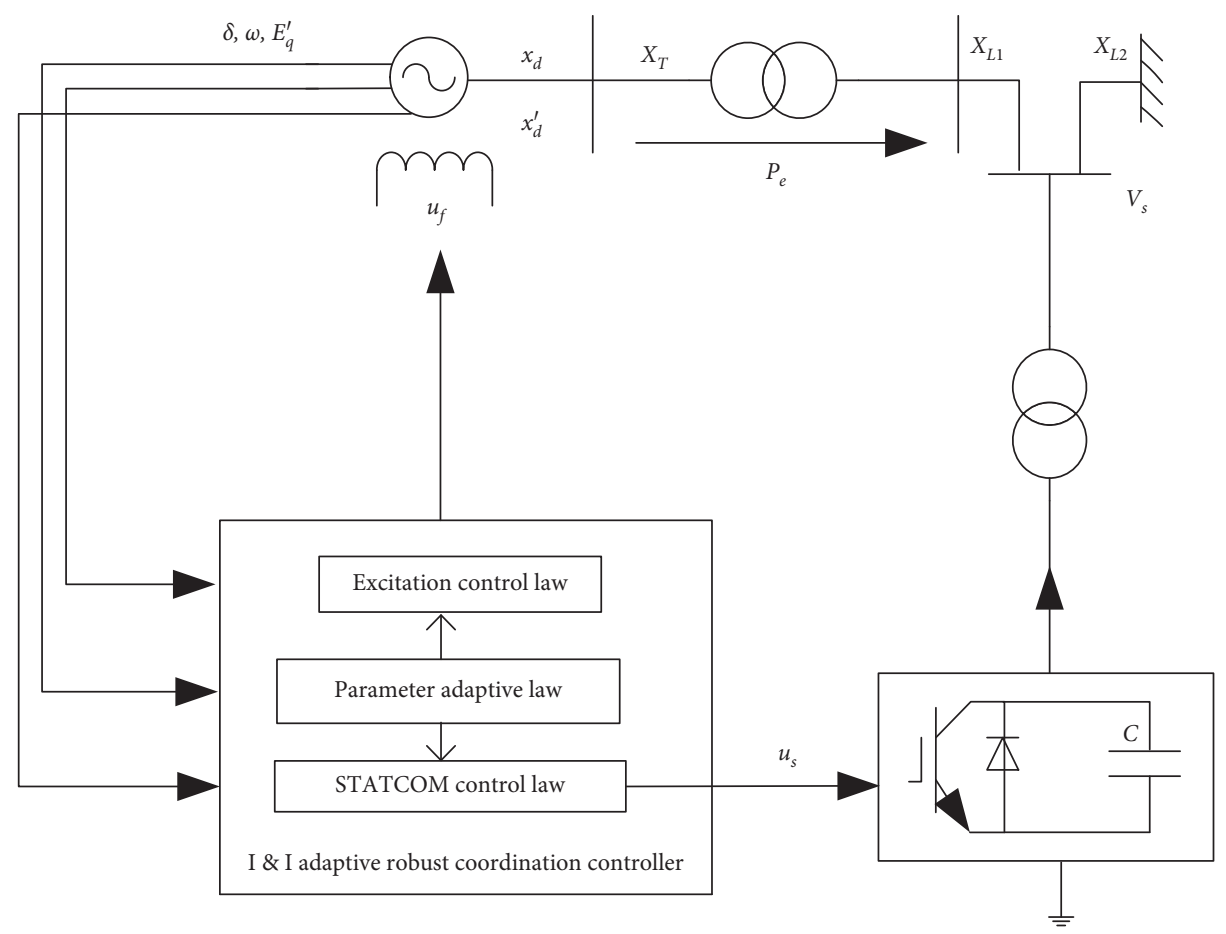

FIgURE 1: Configuration of generator excitation and STATCOM coordinated control system.

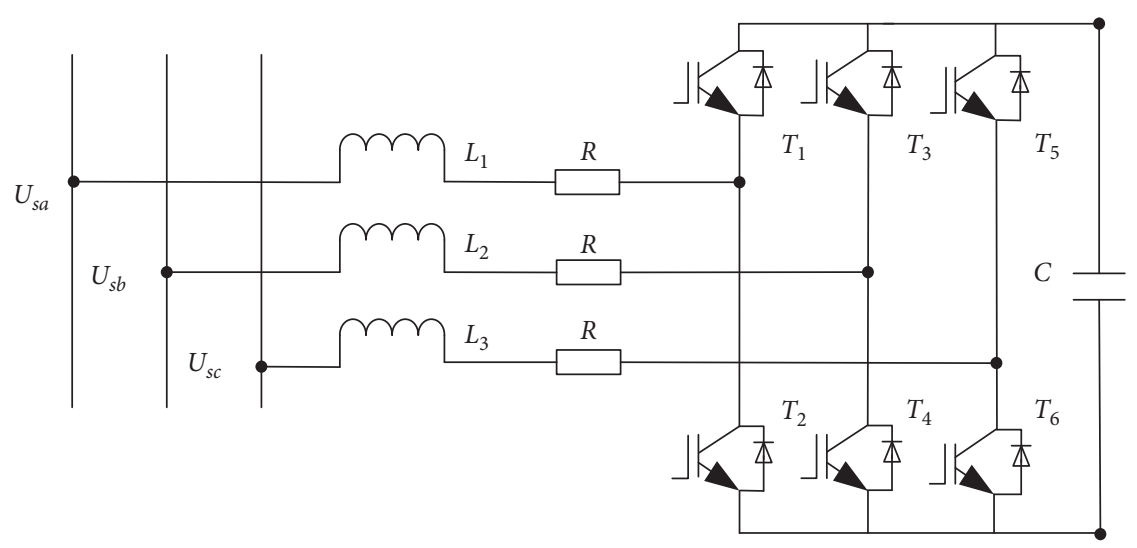

FIGURE 2: Topology structure of voltage-type STATCOM.

Assumption 1. Not considering the effects of the governor.

Assumption 2. Not considering the stator loop resistance and the rotor damping winding.

In case of Assumptions 1 and 2, the generator excitation system studied in this paper adopts the third-order mathematical model. The topology of STATCOM is voltage type shown in Figure 2, and the first-order controllable current source model is adopted. Therefore, the state equation of the generator excitation and STATCOM coordination control system shown in Figure 1 is as follows:

$$
\left\{\begin{array}{l}
\dot{\delta}=\omega-\omega_{0}, \\
\dot{\omega}=-\frac{D}{H}\left(\omega-\omega_{0}\right)+\frac{\omega_{0}}{H}\left(P_{m}-P_{e}\right)+w_{1}, \\
\dot{E}_{q}^{\prime}=-\frac{x_{d \Sigma}}{x_{d \Sigma}^{\prime} T_{d 0}} E_{q}^{\prime}+\frac{x_{d}-x_{d}^{\prime}}{x_{d \Sigma}^{\prime} T_{d 0}} V_{s} \cos \delta+\frac{1}{T_{d 0}} u_{f}+w_{2}, \\
\dot{I}_{q}=\frac{1}{T_{q}}\left(-I_{q}+I_{q 0}+u_{s}\right)+w_{3},
\end{array}\right.
$$


where $\delta$ is the generator rotor angle, $\omega$ is the generator rotor angular speed, $H$ is the inertia constant, $P_{m}$ is the mechanical power, $D$ is the unit damping coefficient; $P_{e}=\left(E_{q}^{\prime} V_{s} \sin \delta\right) /\left(x_{d \Sigma}^{\prime}\right)$ is the delivered electrical power, $E_{q}^{\prime}$ is the generator $q$-axis transient electromotive force, $V_{s}$ is the bus voltage of the infinite bus system, set to 1 p.u, $x_{d \Sigma}=x_{d}+X_{L}+X_{T}$, and $x_{d \Sigma}^{\prime}=x_{d}^{\prime}+X_{L}+X_{T}$.

$x_{d \Sigma}$ and $x_{d \Sigma}^{\prime}$ are the equivalent steady-state reactance and transient reactance of transmission line, respectively. Among, $x_{d}$ and $x_{d}^{\prime}$ are the generator $d$-axis steadystate reactance and transient reactance, respectively. $X_{T}$ is the reactance of the transformer; $X_{L}=X_{L 1}+X_{L 1}, X_{L 1}$ and $X_{L 2}$ are the equivalent transmission line reactance, respectively; $T_{d 0}$ is the open-circuit transient time constant of $d$-axis; $T_{q}$ is the inertial time constant of STATCOM; $I_{q}$ is the output current of the equivalent controllable power supply of STATCOM; $u_{f}$ and $u_{s}$ are the control input of the generator excitation and STATCOM, respectively. $w=\left[\begin{array}{lll}w_{1} & w_{2} & w_{3}\end{array}\right]^{T}$ is the uncertainty disturbance applied to the generator rotor, admittance, and STATCOM controller, and $\left|w_{i}(t)\right| \leq h_{i}$ holds, and $h_{i}$ is a positive number.

In the coordinated control system, select the state variables of the system as $\left[\begin{array}{llll}x_{1} & x_{2} & x_{3} & x_{4}\end{array}\right]^{T}=$ $\left[\begin{array}{llll}\delta-\delta_{0} & \omega-\omega_{0} & I_{q}-I_{q 0} & E_{q}^{\prime}-E_{q 0}^{\prime}\end{array}\right]^{T}$, where the initial values correspond to the variables of $\delta_{0}, \omega_{0}, I_{q 0}$, and $E_{q 0}^{\prime}$. Assume that the input is $u_{s}$ and $u_{f}$, the output $y=\Delta E_{q}^{\prime}$, $\theta_{1}=-D / H$ and $\theta_{2}=P_{m}$, and $\theta_{1}$ and $\theta_{2}$ is the uncertain parameter. The system model (8) can be manipulated as

$$
\left\{\begin{array}{l}
\dot{x}_{1}=x_{2} \\
\dot{x}_{2}=\theta_{1} x_{2}+a_{1} \theta_{2}-a_{1} \Delta P_{e}+w_{1} \\
\dot{x}_{3}=a_{2} x_{3}+a_{3} u_{s}+w_{3} \\
\dot{y}=a_{4} y+a_{5} \cos \left(x_{1}+\delta_{0}\right)+a_{6} u_{f}+w_{2}
\end{array}\right.
$$

where $\quad a_{1}=\omega_{0} / H, \quad a_{2}=-1 / T_{q}, \quad a_{3}=1 / T_{q} a_{4}=-x_{d \Sigma} /$ $\left(T_{d 0} x_{d \Sigma}^{\prime}\right), a_{5}=\left(\left(x_{d \Sigma}-x_{d \Sigma}^{\prime}\right) V_{s}\right) /\left(T_{d 0} x_{d \Sigma}^{\prime}\right), a_{6}=1 / T_{d 0}, \quad b=$ $\left(-x_{d \Sigma} E_{q 0}^{\prime}\right) /\left(T_{d 0} x_{d \Sigma}^{\prime}\right), \quad \Delta P_{e}=\left(\left(\left(y+E_{q 0}^{\prime}\right) V_{s} \sin \left(x_{1}+\delta_{0}\right)\right) /\right.$ $\left.\left(x_{d \Sigma}^{\prime}+\Delta x\right)\right)-\left(E_{q 0}^{\prime} V_{s} \sin \delta_{0} / x_{d \Sigma}^{\prime}\right)$, and $\Delta x=X_{L 1} X_{L 2} \Delta I_{q}$.

To facilitate the design of the controller, (9) can be written as follows:

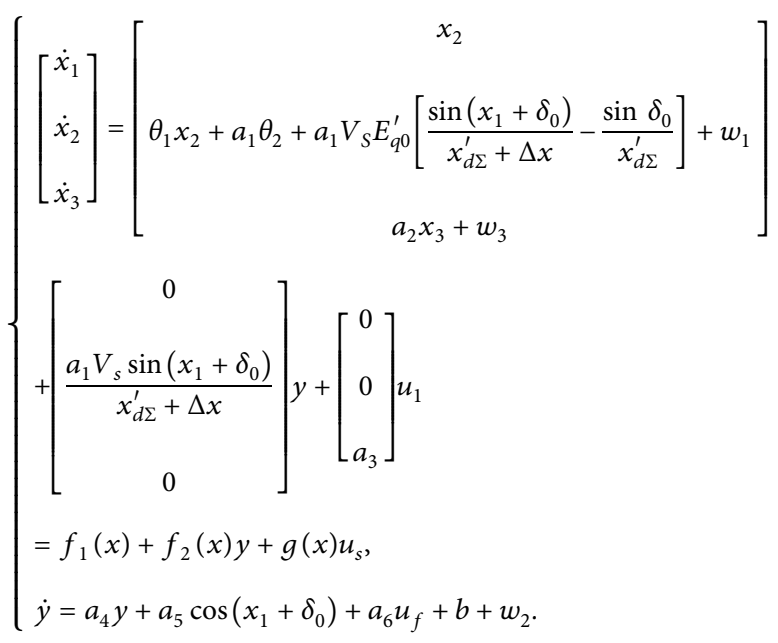

Control objective is as follows. An I\&I adaptive robust coordinated control strategy is designed for system (8) with multiple parameter uncertainties and unknown disturbances. Design the adaptive laws to recognize the uncertain parameters. Design the coordinated control laws for the generator excitation and the STATCOM so that the control system is robust for the uncertain disturbances and the error system has a globally asymptotical stability at the equilibrium point which indicates that the state error variables converge to zero.

\section{Controller Design}

The design process of generator excitation and STATCOMcoordinated controller is mainly as follows: under the condition of no excitation control, aiming at the coordinated control system (2) with uncertain parameters, damping coefficient, and mechanical power. Firstly, the I\&I control method is adopted to design the uncertain parameter adaptive law; then, the STATCOM control law is designed by using the backstepping method and nonlinear damping algorithm. Applying passivity theory, the excitation control law is designed.

According to the idea of passivity (9), the state equation without excitation control is

$$
\left\{\begin{array}{l}
\dot{x}_{1}=x_{2}, \\
\dot{x}_{2}=\theta_{1} x_{2}+a_{1} \theta_{2}-a_{1} \Delta P_{e 1}+w_{1}, \\
\dot{x}_{3}=a_{2} x_{3}+a_{3} u_{s}+w_{3},
\end{array}\right.
$$

where $\quad \Delta P_{e 1}=\left(\left(\left(y+E_{q 0}^{\prime}\right) V_{s} \sin \left(x_{1}+\delta_{0}\right)\right) /\left(x_{d \Sigma}^{\prime}+\Delta x\right)\right)$ $-\left(\left(E_{q 0}^{\prime} V_{s} \sin \delta_{0}\right) / x_{d \Sigma}^{\prime}\right)$.

4.1. Parameter Adaptive Law Design. According to the I\&I adaptive control method, an adaptive law is designed for two uncertain parameters at the same time. The process is as follows.

Firstly, the parameter estimation errors of two uncertain parameters are defined as follows:

$$
\left\{\begin{array}{l}
z_{1}=\widehat{\theta}_{1}-\theta_{1}+\beta_{1}\left(x_{1}, x_{2}\right), \\
z_{2}=\widehat{\theta}_{2}-\theta_{2}+\beta_{2}\left(x_{1}, x_{2}\right),
\end{array}\right.
$$

where $\widehat{\theta}_{1}$ and $\widehat{\theta}_{2}$ are estimates of $\theta_{1}$ and $\theta_{2}$, respectively, and $\beta_{1}\left(x_{1}, x_{2}\right)$ and $\beta_{2}\left(x_{1}, x_{2}\right)$ are the functions to be designed, respectively.

Then, by substituting (11) into (12), therefore, (12) is obtained as

$$
\left\{\begin{array}{l}
\dot{z}_{1}=\dot{\hat{\theta}}_{1}+\sum_{k=1}^{2} \frac{\partial \beta_{1}}{\partial x_{k}} \times \dot{x}_{k} \\
=\dot{\hat{\theta}}_{1}+\frac{\partial \beta_{1}}{\partial x_{1}} \dot{x}_{1}+\frac{\partial \beta_{1}}{\partial x_{2}}\left[\theta_{1} x_{2}+a_{1} \theta_{2}-a_{1} \Delta P_{e 1}+w_{1}\right] \\
\dot{z}_{2}=\dot{\hat{\theta}}_{2}+\sum_{k=1}^{2} \frac{\partial \beta_{2}}{\partial x_{k}} \times \dot{x}_{k} \\
=\dot{\hat{\theta}}_{2}+\frac{\partial \beta_{2}}{\partial x_{1}} \dot{x}_{1}+\frac{\partial \beta_{2}}{\partial x_{2}}\left[\theta_{1} x_{2}+a_{1} \theta_{2}-a_{1} \Delta P_{e 1}+w_{1}\right] .
\end{array}\right.
$$


The adaptive laws for designing two uncertain parameters are as follows:

$$
\left\{\begin{array}{l}
\dot{\hat{\theta}}_{1}=-\frac{\partial \beta_{1}}{\partial x_{1}} x_{2}-\frac{\partial \beta_{1}}{\partial x_{2}}\left[\left(\hat{\theta}_{1}+\beta_{1}\right) x_{2}+a_{1}\left(\widehat{\theta}_{2}+\beta_{2}\right)-a_{1} \Delta P_{e 1}-h_{1}\right] \\
\dot{\hat{\theta}}_{2}=-\frac{\partial \beta_{2}}{\partial x_{1}} x_{2}-\frac{\partial \beta_{2}}{\partial x_{2}}\left[\left(\hat{\theta}_{1}+\beta_{1}\right) x_{2}+a_{1}\left(\hat{\theta}_{2}+\beta_{2}\right)-a_{1} \Delta P_{e 1}-h_{1}\right] .
\end{array}\right.
$$

Substituting (14) into (13), we can obtain

$$
\left\{\begin{array}{l}
\dot{z}_{1}=-\frac{\partial \beta_{1}}{\partial x_{2}}\left(z_{1} x_{2}+z_{2} a_{1}-\left(h_{1}-w_{1}\right)\right) \\
\dot{z}_{2}=-\frac{\partial \beta_{2}}{\partial x_{2}}\left(z_{1} x_{2}+z_{2} a_{1}-\left(h_{1}-w_{1}\right)\right)
\end{array}\right.
$$

Take the adjustable functions as follows:

$$
\left\{\begin{array}{l}
\beta_{1}\left(x_{1}, x_{2}\right)=\rho x_{2}^{2} \\
\beta_{2}\left(x_{1}, x_{2}\right)=\rho a_{1} x_{2}
\end{array}\right.
$$

where $\rho>0$.

Construct the parameter estimation error system (15) with the alternative Lyapunov function (CLF) as

$$
V\left(z_{1}, z_{2}\right)=\frac{1}{2} z_{1}^{2}+\frac{1}{2} z_{2}^{2}
$$

The derivative of $V\left(z_{1}, z_{2}\right)$ is

$$
\begin{aligned}
\dot{V}\left(z_{1}, z_{2}\right)= & z_{1} \dot{z}_{1}+z_{2} \dot{z}_{2} \\
= & -\frac{\partial \beta_{1}}{\partial x_{2}}\left[\left(z_{1} x_{2}+z_{2} a_{1}\right)-\left(h_{1}-w_{1}\right)\right] z_{1} \\
& -\frac{\partial \beta_{2}}{\partial x_{2}}\left[\left(z_{1} x_{2}+z_{2} a_{1}\right)-\left(h_{1}-w_{1}\right)\right] z_{2} \\
\leq & -\rho\left[\left(z_{1} x_{2}+z_{2} k_{1}\right)-\frac{\left(h_{1}-w_{1}\right)}{2}\right]^{2} \leq 0 .
\end{aligned}
$$

According to (18), $V\left(z_{1}, z_{2}\right)$ is negative under the parameter adaptive law (14). Therefore, according to LaSalle's theorem, the designed parameter adaptive law can ensure the asymptotic stability of the dynamics of the parameter estimation error.

\subsection{Design Control Law of STATCOM $u_{1}$}

Step 1 : the state error function of the system is defined as

$$
\begin{aligned}
& e_{1}=x_{1}, \\
& e_{2}=x_{2}-x_{2}^{*},
\end{aligned}
$$

where $x_{2}^{*}$ is "virtual control quantity" and its control law will be designed in the following backstepping method.
Step 2 : firstly, the derivation of $e_{1}$ is given by

$$
\dot{e}_{2}=\theta x_{2}+k_{1} P_{m}-k_{2} \frac{\left(x_{3}+E_{q 0}^{\prime}\right)}{x_{d \Sigma}^{\prime}} \sin \left(x_{1}+\delta_{0}\right)+w_{1}+c_{1} x_{2}
$$

According to the idea of document [19], $e_{2}$ is regarded as an uncertain term and Assumption 1: $\left|e_{2}\right| \leq \chi_{1}$, where $\chi_{1}$ is the normal number to be designed.

Applying the nonlinear damping algorithm, $x_{2}^{*}$ is designed as follows:

$$
x_{2}^{*}=-k_{1} e_{1}-\frac{\chi_{1}^{2} e_{1}}{\left(2 \lambda_{1}\right)},
$$

where $k_{1}>0, \lambda_{1}>0$, and $\chi_{1}^{2} e_{1} /\left(2 \lambda_{1}\right)$ is the nonlinear damping term to compensate for uncertainties [19].

Take the Lyapunov function of (11) as

$$
V_{1}=\frac{1}{2} e_{1}^{2}
$$

Then, the derivation of $V_{1}$ can be described as

$$
\begin{aligned}
\dot{V}_{1} & =e_{1} \dot{e}_{1}=e_{1}\left(e_{2}+x_{2}^{*}\right)=e_{1} e_{2}-k_{1} e_{1}^{2}-\frac{\chi_{1}^{2} e_{1}^{2}}{\left(2 \lambda_{1}\right)} \\
& =-k_{1} e_{1}^{2}-\frac{\chi_{1}^{2} e_{1}^{2}}{\left(2 \lambda_{1}\right)}+\left|e_{1}\right|\left|e_{2}\right| \\
& \leq-k_{1} e_{1}^{2}+\chi_{1}\left|e_{1}\right|-\frac{\chi_{1}^{2} e_{1}^{2}}{\left(2 \lambda_{1}\right)} .
\end{aligned}
$$

When $\left|e_{1}\right| \geq 2 \lambda_{1} / \chi_{1}$ is satisfied, we have

$$
\dot{V}_{1} \leq-k_{1} e_{1}^{2} \leq 0
$$

Step 3 : the derivation of $e_{2}$ is given by

$$
\begin{aligned}
\dot{e}_{2} & =\dot{x}_{2}-\dot{x}_{2}^{*}=\theta_{1} x_{2}+a_{1} \theta_{2}-a_{1} \Delta P_{e 1}+w_{1}-\dot{x}_{2}^{*} \\
& =\theta_{1} x_{2}+a_{1} \theta_{2}-a_{1} \Delta P_{e d}+a_{1} e_{3}+w_{1}-\dot{x}_{2}^{*} .
\end{aligned}
$$

Similarly, according to the idea of [19], $a_{1} e_{3}-\dot{x}_{2}^{*}$ is regarded as an uncertain term and Assumption 2: $\left|a_{1} e_{3}+w_{1}-\dot{x}_{2}^{*}\right| \leq \chi_{2}$, where $\chi_{2}$ is the normal number to be designed.

Applying the nonlinear damping algorithm, $\Delta P_{e 1 d}$ is designed as follows:

$$
\Delta P_{e 1 d}=\frac{1}{a_{1}}\left[\left(\hat{\theta}_{1}+\frac{1}{2} x_{2}^{2}\right) x_{2}+\left(\hat{\theta}_{2}+a_{1} x_{2}\right)+k_{2} e_{2}+\frac{\chi_{2}^{2} e_{2}}{2 \lambda_{2}}\right]
$$

where $k_{2}>0, \lambda_{2}>0$, and $\chi_{2}^{2} e_{2} /\left(2 \lambda_{2}\right)$ is the nonlinear damping term to compensate for uncertainties [19].

The extended Lyapunov function is 


$$
V_{2}=V_{1}+\frac{1}{2} e_{2}^{2}
$$

Then, the derivation of $V_{2}$ can be described as

$$
\begin{aligned}
\dot{V}_{2} & =\dot{V}_{1}+e_{2} \dot{e}_{2}=-k_{1} e_{1}^{2}+e_{2}\left(\theta_{1} x_{2}+a_{1} \theta_{2}-a_{1} \Delta P_{e 1 d}+a_{1} e_{3}+w_{1}-\dot{x}_{2}^{*}\right) \\
& =-k_{1} e_{1}^{2}-k_{2} e_{2}^{2}-e_{2}\left(z_{1} x_{2}+z_{2} a_{1}\right)+\left|e_{2}\right|\left|a_{1} e_{3}+w_{1}-\dot{x}_{2}^{*}\right|-\frac{\chi_{2}^{2} e_{2}^{2}}{\left(2 \lambda_{2}\right)} \\
& \leq-k_{1} e_{1}^{2}-k_{2} e_{2}^{2}-e_{2}\left(z_{1} x_{2}+z_{2} a_{1}\right)+\chi_{2}\left|e_{2}\right|-\frac{\chi_{2}^{2} e_{2}^{2}}{\left(2 \lambda_{2}\right)} .
\end{aligned}
$$

According to the abovementioned I\&I parameter estimation error, derivative dynamics is asymptotically stable; then, $\lim _{z_{i=12} \longrightarrow \infty} e_{2}\left(z_{1} x_{2}+z_{2} a_{1}\right)=0$ holds.

When $\left|e_{2}\right| \geq 2 \varepsilon_{2} / \chi_{2}$ is satisfied, we have

$$
\dot{V}_{2} \leq-k_{1} e_{1}^{2}-k_{2} e_{2}^{2}-e_{2}\left(z_{1} x_{2}+z_{2} a_{1}\right) \leq 0 .
$$

Step 4 : define $e_{3}=\Delta P_{e d}-\Delta P_{e 1 d}$, and set $\Delta P_{e 1 d}$ as the virtual control quantity.

The derivation of $e_{3}$ is given by

$$
\begin{aligned}
\dot{e}_{3}= & \Delta \dot{P}_{e}-\Delta \dot{P}_{e d} \\
= & c_{1} \cos \left(x_{1}+\delta_{0}\right) x_{2}-c_{2} \sin \left(x_{1}+\delta_{0}\right)\left(a_{2} x_{3}+a_{3} u_{s}+w_{3}\right) \\
= & c_{1} \cos \left(x_{1}+\delta_{0}\right) x_{2}-c_{2} \sin \left(x_{1}+\delta_{0}\right)\left(a_{2} x_{3}+a_{3} u_{s}\right) \\
& -\left[\Delta \dot{P}_{e 1 d}+c_{2} \sin \left(x_{1}+\delta_{0}\right) w_{3}\right],
\end{aligned}
$$

where $c_{1}=\left(E_{q 0}^{\prime} V_{s}\right) / x_{d \Sigma}^{\prime}$ and $c_{2}=\left(E_{q 0}^{\prime} V_{s} x_{L 1} x_{L 2}\right) / x_{d \Sigma}^{\prime 2}$.

Similarly, $-\Delta \dot{P}_{e 1 d}-c_{2} \sin \left(x_{1}+\delta_{0}\right) w_{3}$ is regarded as an uncertain term, and according to idea of [19], $\left|-\Delta \dot{P}_{e 1 d}-c_{2} \sin \left(x_{1}+\delta_{0}\right) w_{3}\right| \leq \chi_{3}$, where $\chi_{3}$ is the normal number to be designed.

Applying the nonlinear damping algorithm, when $\sin \left(x_{1}+\delta_{0}\right) \neq 0, u_{s}$ is designed as follows:

$$
\begin{array}{r}
u_{s}=\frac{1}{a_{3} c_{2} \sin \left(x_{1}+\delta_{0}\right)}\left[c_{1} x_{2} \cos \left(x_{1}+\delta_{0}\right)-\right. \\
\left.a_{2} c_{2} \sin \left(x_{1}+\delta_{0}\right)+k_{3} e_{3}+\frac{\chi_{3}^{2} e_{3}}{\left(2 \lambda_{3}\right)}\right] .
\end{array}
$$

Take the global Lyapunov function of (11) as follows:

$$
V_{3}=V_{2}+\frac{1}{2} e_{3}^{2}
$$

Then, the derivation of $V_{3}$ can be described as

$$
\begin{aligned}
\dot{V}_{3}= & \dot{V}_{2}+e_{3} \dot{e}_{3} \\
= & \dot{V}_{2}+e_{3}\left[c_{1} \cos \left(x_{1}+\delta_{0}\right) x_{2}\right. \\
& \left.-c_{2} \sin \left(x_{1}+\delta_{0}\right)\left(a_{2} x_{3}+a_{3} u_{s}+w_{3}\right)-\Delta \dot{P}_{e 1 d}\right] \\
= & -k_{1} e_{1}^{2}-k_{2} e_{2}^{2}-e_{2}\left(z_{1} x_{2}+z_{2} a_{1}\right) \\
& -k_{3} e_{3}^{2}+\left|e_{3}\right|\left|-\Delta \dot{P}_{e 1 d}-c_{2} \sin \left(x_{1}+\delta_{0}\right) w_{3}\right|-\frac{\chi_{3}^{2} e_{3}^{2}}{\left(2 \lambda_{3}\right)} \\
\leq & -k_{1} e_{1}^{2}-k_{2} e_{2}^{2}-e_{2}\left(z_{1} x_{2}+z_{2} a_{1}\right)-k_{3} e_{3}^{2}+\chi_{3}\left|e_{2}\right|-\frac{\chi_{3}^{2} e_{3}^{2}}{\left(2 \lambda_{3}\right)} .
\end{aligned}
$$

When $\left|e_{3}\right| \geq 2 \lambda_{3} / \chi_{3}$ is satisfied, we have

$$
\dot{V}_{3} \leq-k_{1} e_{1}^{2}-k_{2} e_{2}^{2}-k_{3} e_{3}^{2}-e_{2}\left(z_{1} x_{2}+z_{2} a_{1}\right) \text {. }
$$

4.3. Design the Control Law of Generator Excitation $u_{2}$. Let $W(e)=V_{3}$, and take the storage function of the whole system as

$$
V=W(e)+\frac{1}{2} y^{2}
$$

According to equation (5), $u_{2}$ is designed as

$$
u_{f}=\frac{1}{a_{6}}\left[\frac{e_{2} \omega_{0} V_{s}}{H x_{d \Sigma}^{\prime}} \sin \left(x_{1}+\delta_{0}\right)-a_{5} \cos \left(x_{1}+\delta_{0}\right)-b+v\right] .
$$

Then, the derivation of $V$ can be described as

$$
\begin{aligned}
\dot{V} & =\left.\frac{\partial W(e)}{\partial e} \dot{e}\right|_{y=0}+y \dot{y} \\
& =\left.\frac{\partial W(e)}{\partial e} \dot{e}\right|_{y=0}+a_{4} y^{2}+v y \\
& \leq a_{4} y^{2}+v y \leq v y .
\end{aligned}
$$


Due to $\dot{V} \leq v y$, the passivity condition is satisfied. In addition, take $v=-k_{4} y$, where $k_{4}>0$. According to Theorem 1 , system (8) can be guaranteed to be asymptotically stable at the equilibrium.
To sum up, the control law of the generator and STATCOM coordination controller is as followed:

$$
\left\{\begin{array}{l}
u_{s}=\frac{1}{a_{3} c_{2} \sin \left(x_{1}+\delta_{0}\right)}\left[c_{1} x_{2} \cos \left(x_{1}+\delta_{0}\right)-a_{2} c_{2} \sin \left(x_{1}+\delta_{0}\right)+k_{3} e_{3}+\frac{\chi_{3}^{2} e_{3}}{\left(2 \lambda_{3}\right)}\right] \\
u_{f}=\frac{1}{a_{6}}\left[\frac{e_{2} \omega_{0} V_{s}}{H x_{d \Sigma}^{\prime}} \sin \left(x_{1}+\delta_{0}\right)-a_{5} \cos \left(x_{1}+\delta_{0}\right)-b+v\right]
\end{array}\right.
$$

Therefore, the closed-loop error system of the system (equation (8)) is

$$
\left\{\begin{array}{l}
\dot{e}_{1}=e_{2}-k_{1} e_{1}-\frac{\chi_{1}^{2} e_{1}}{\left(2 \lambda_{1}\right)}, \\
\dot{e}_{2}=-\rho\left(z_{1} x_{2}+z_{2} a_{1}\right)-k_{2} e_{2}-\frac{\chi_{2}^{2} e_{2}}{\left(2 \lambda_{2}\right)}, \\
\dot{e}_{3}=-k_{3} e_{3}-\frac{\chi_{3}^{2} e_{3}}{\left(2 \lambda_{3}\right)}, \\
\dot{y}=a_{4} y-\frac{e_{2} a_{1} V_{s} \sin \left(x_{1}+\delta_{0}\right)}{x_{d \Sigma}^{\prime}}+v .
\end{array}\right.
$$

According to formula (37), the state error variables of the closed-loop system (39) converge to zero. Therefore, the state variables of system (8) are stable at the equilibrium point.

Compared with the adaptive backstepping coordinated control method adopted in [15], the advantages are as follows. (1) Based on the I\&I adaptive control method, the adaptive laws of two uncertain parameters in the system are designed, and two tunable functions are added to the adaptive laws, which improves the flexibility and control ability of parameter estimation. (2) The design of adaptive laws is separated from the design of control laws, and the nonlinear damping term is introduced in the backstepping method to compensate the uncertain term, which solves the problem of "calculation expansion" to a certain extent.

\section{Simulation Results and Analysis}

MATLAB is adopted for simulation analysis. We construct the simulation system model represented by formula (8) with $S$ function. And, the system simulation parameters are as follows [14, 17]: $H=5.9, E_{q}^{\prime}=1.0, \omega_{0}=314.15, \delta_{0}=50^{\circ}$, $T_{q}=0.02, \quad T_{d 0}=0.59, \quad x_{d}=1.5, \quad x_{d}^{\prime}=0.5, \quad X_{L 1}=0.2$, $X_{L}=2.2 X_{L 2}=0.1$, and $X_{T}=0.7$. The virtual control variable parameters are as follows: $k_{1}=200, k_{2}=10, k_{3}=20$, $k_{4}=450, \chi_{1}=6, \chi_{2}=20, \chi_{3}=0.1, \lambda_{1}=0.55, \lambda_{2}=20$, and $\lambda_{3}=0.02$. Under the same conditions, the design in this paper and the adaptive backstepping method in [15] are divided into two cases for simulation analysis. Among them, the simulation diagrams of STATCOM control input $u_{\mathrm{s}}$ and excitation control input $u_{f}$ within the physical change range are shown in Figures 3 and 4.

5.1. Simulation Analysis under Small Interference. When $t=0$, the power angle of the generator deviates from the equilibrium point, which indicates that the system has suffered small interference. Figures 5-8 show the simulation results of generator power angle, generator angular velocity, generator potential, and equivalent current of STATCOM. In the figure, the solid line is the simulation result under the control of the improved adaptive backstepping passive coordinated control (IABPC) proposed in this paper, and the dashed line is the adaptive backstepping coordinated control (ABCC) used in the literature's [15] simulation result.

It can be seen from Figures 5-8 that when the system suffers from small interference, compared with the ABCC control method, the IABPC control method can quickly quell the power oscillation, and the time for restoring stability is shortened, which proves that IABPC controller has better static performance.

5.2. Simulation Analysis under the Condition of Large Interference. When $t=0.1$, three-phase short circuit occurs at the outlet of the bus at the high voltage side of the transformer, and the fault is removed in $0.15 \mathrm{~s}$. The simulation results are shown in Figures 9-12. It can be seen from Figures 9-12 that when the system is greatly disturbed, the IABPC control method can quickly stabilize the disturbed system and make it return to a stable state with fast convergence speed and small oscillation range and amplitude, which improves the transient stability of the system. Its effect is better than that of the ABCC control method.

To verify the strong adaptive ability of the controller designed in this paper, the IABPC method and the ABCC method are compared and analyzed, and the simulation results are shown in Figures 13-14. In the simulation, if the damping coefficient $D=1$, the parameter $=-0.143$; if the damping coefficient $\mathrm{Pm}=1$, the parameter $=1$. From 


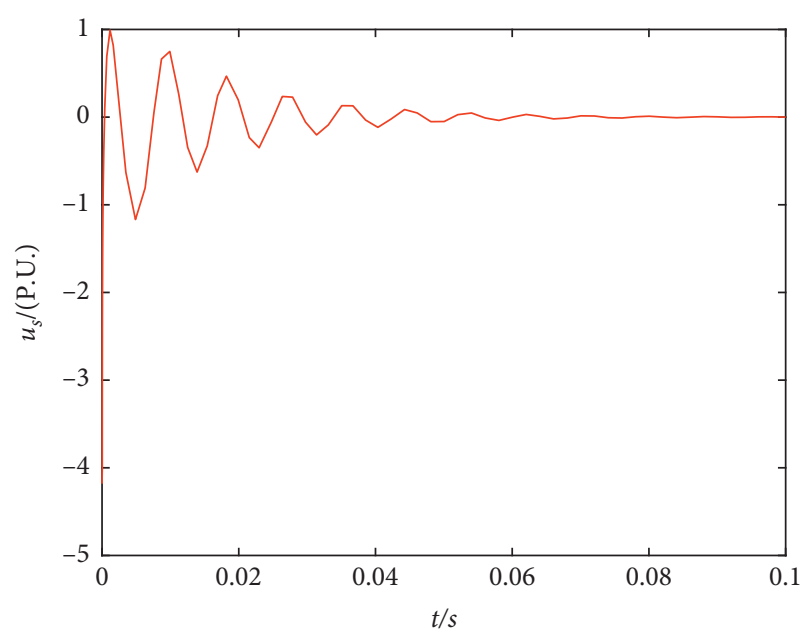

FIgURE 3: Control input of $u_{s}$.

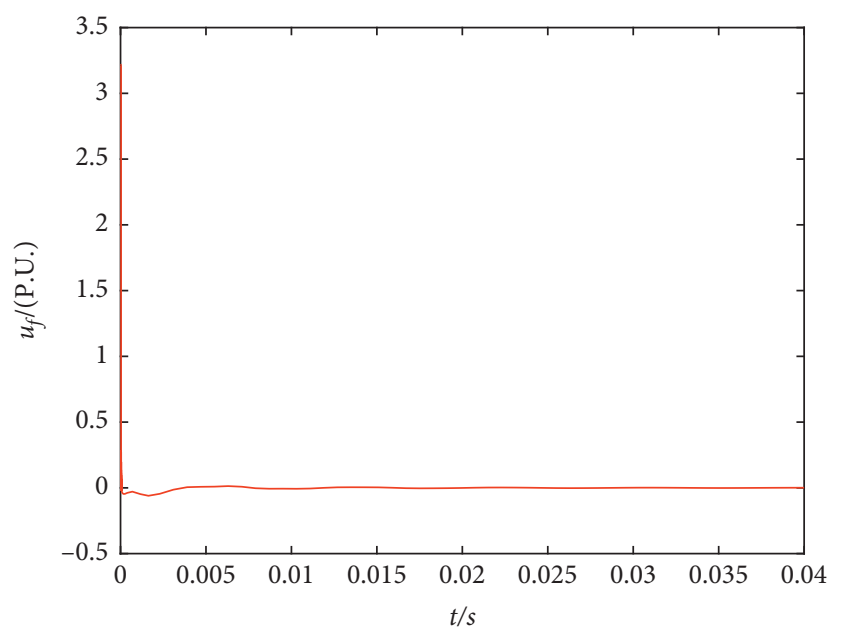

FIGURE 4: Control input of $u_{f}$.

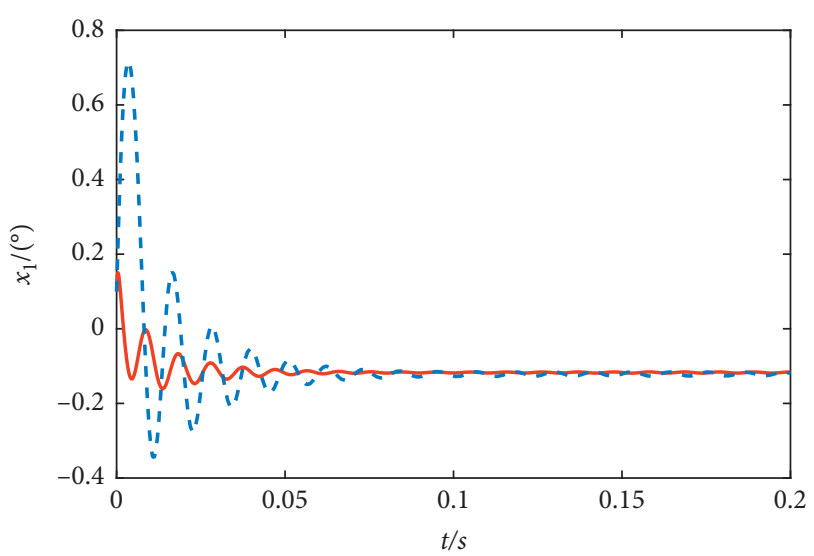

IABPC

- - ABCC

FIgURE 5: Transient response curve of state variable $x_{1}$.

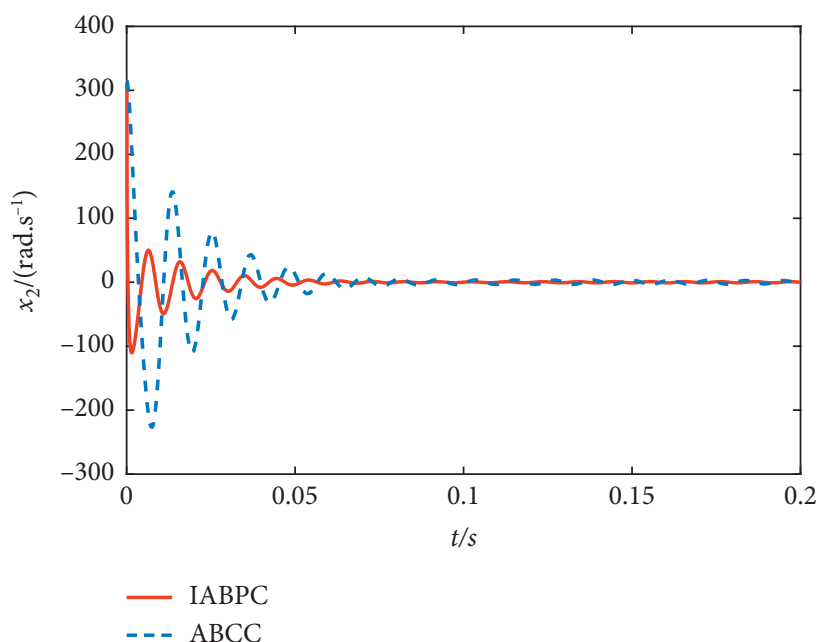

Figure 6: Transient response curve of state variable $x_{2}$.

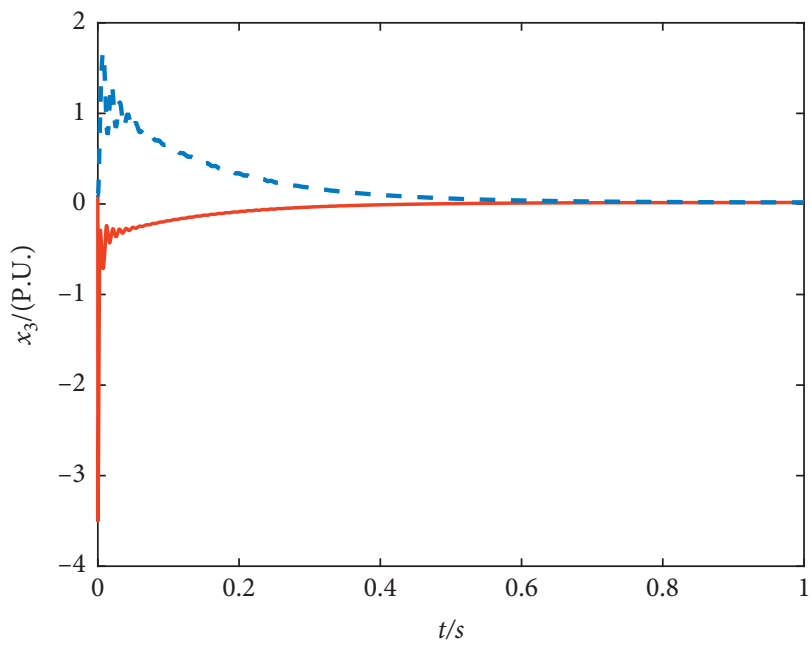

Figure 7: Transient response curve of state variable $x_{3}$.

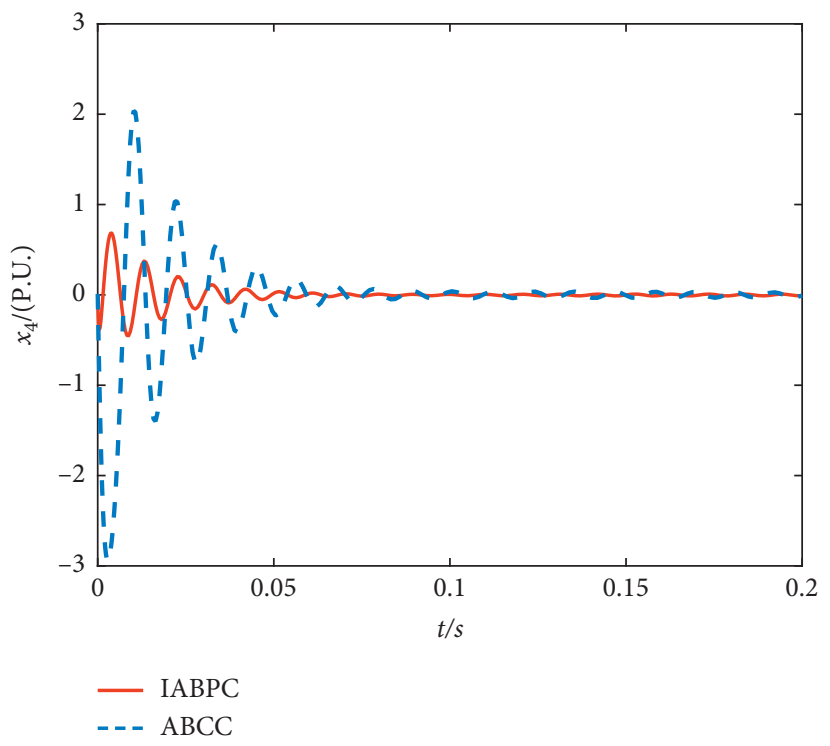

FIgURE 8: Transient response curve of state variable $x_{4}$. 


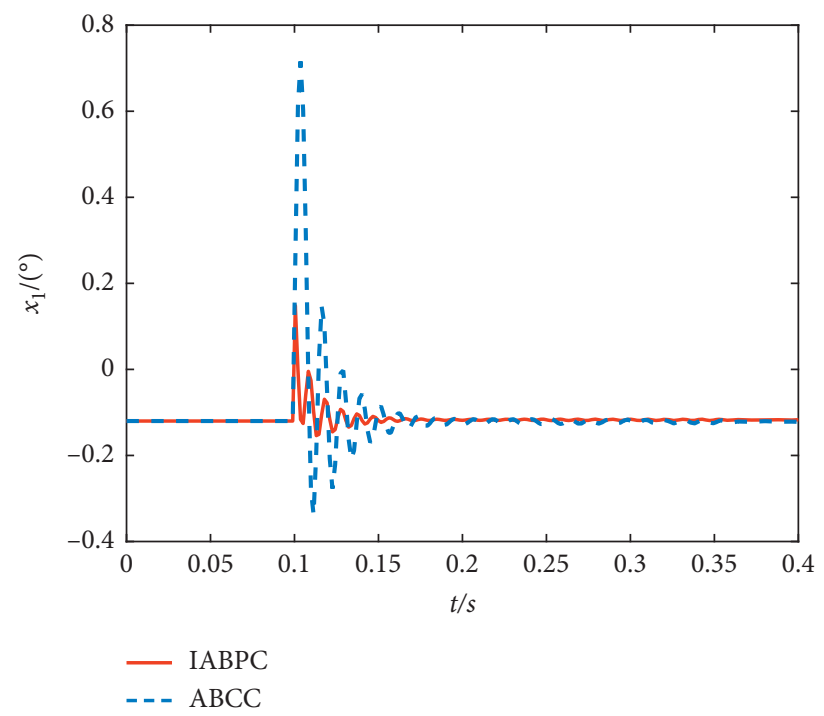

Figure 9: Transient response curve of state variable $x_{1}$.

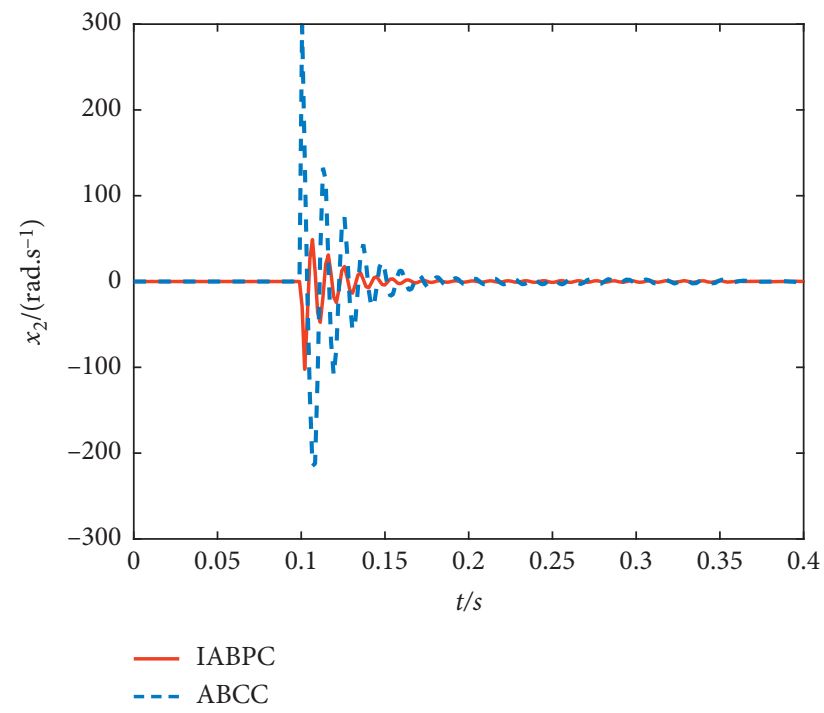

Figure 10: Transient response curve of state variable $x_{2}$.

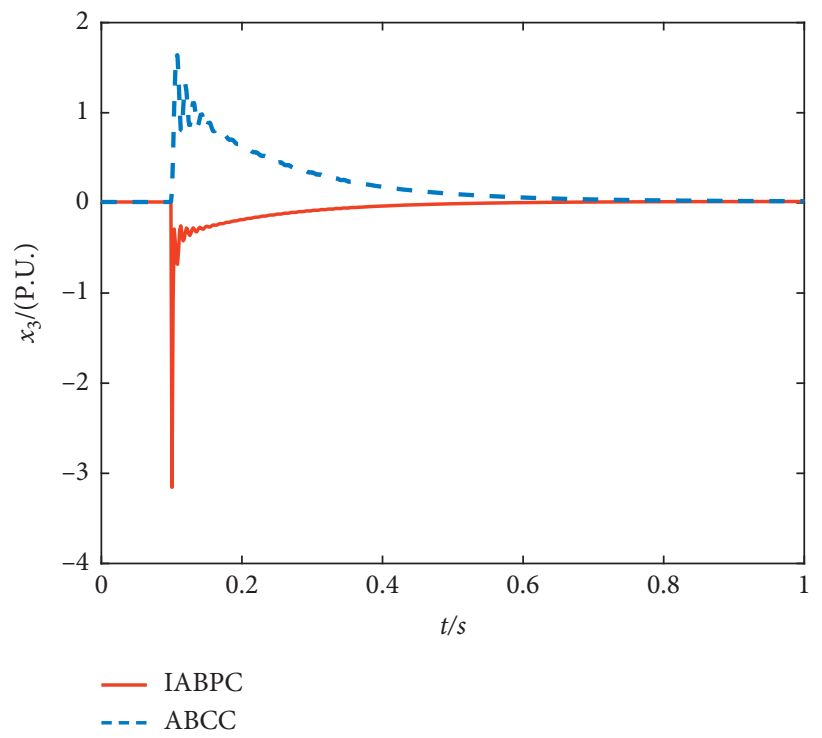

FIgURE 11: Transient response curve of state variable $x_{3}$. 


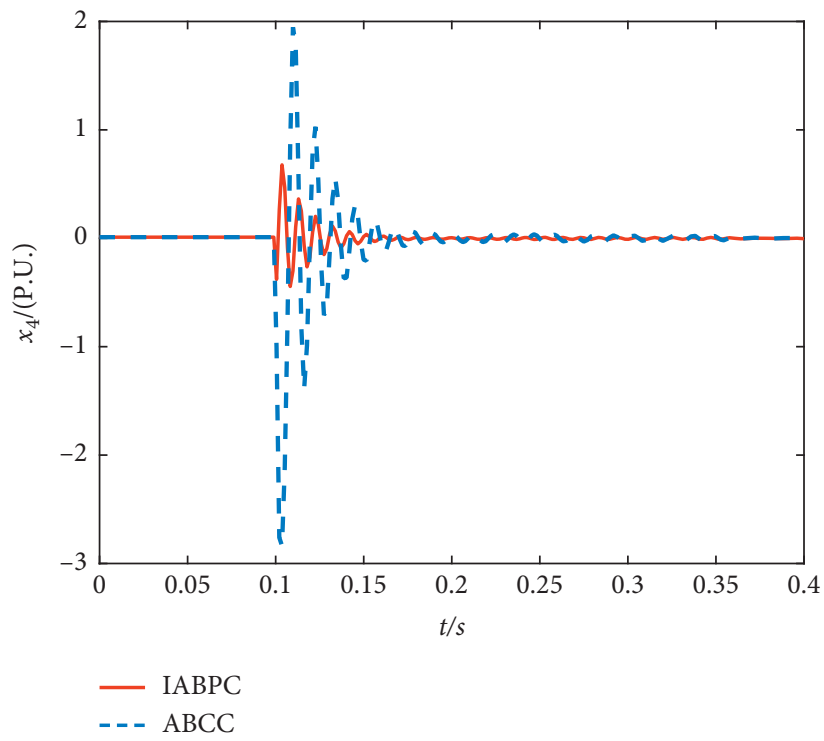

Figure 12: Transient response curve of state variable $x_{4}$.

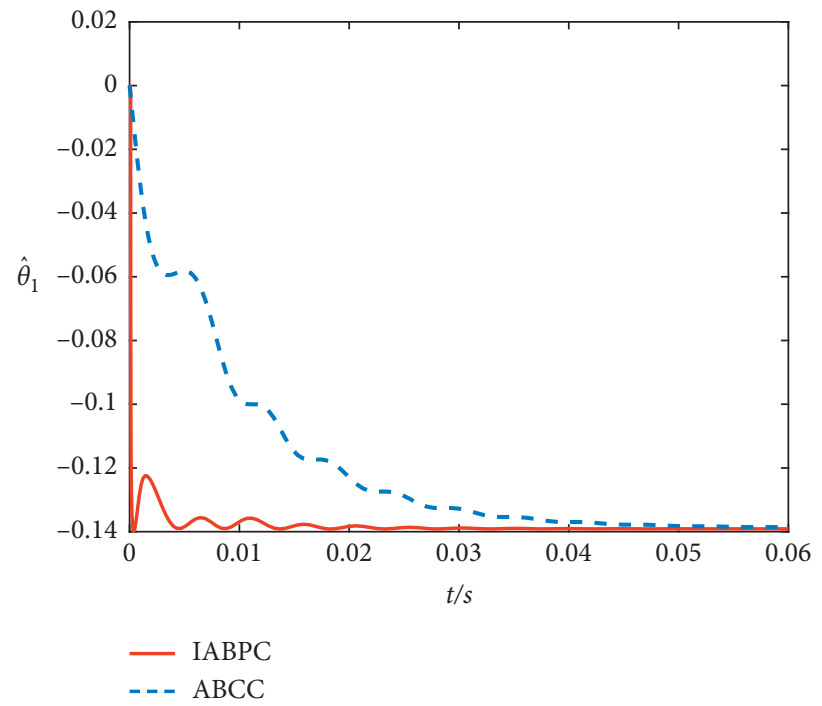

FIGURE 13: Response of uncertain parameter estimation $\theta_{1}$.

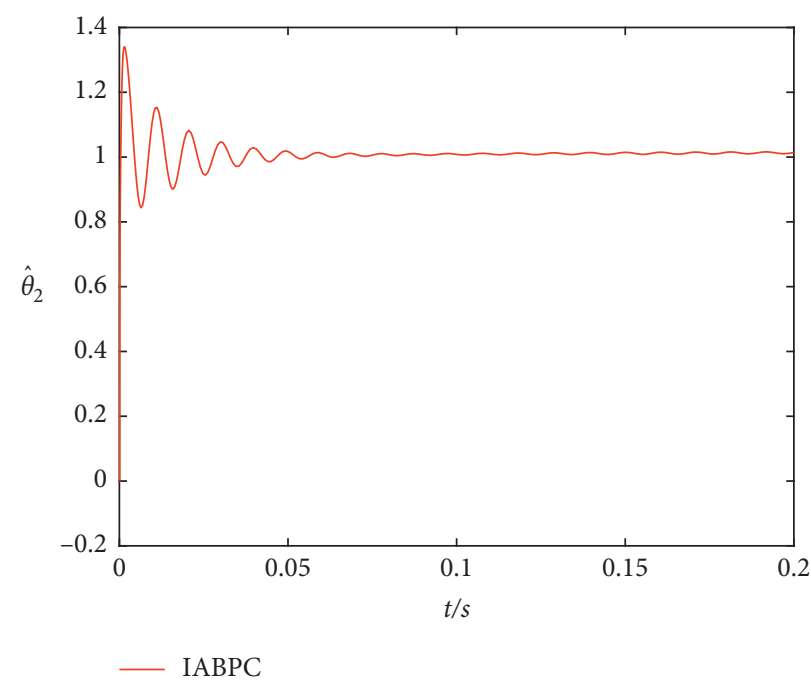

FIGURE 14: Response of uncertain parameter estimation $\theta_{2}$. 
Figures 13 and 14, it can be seen that compared with the ABCC method, the adaptive estimation law designed by the IABPC method is closer to the set value and has stronger adaptive ability.

\section{Conclusion and Future Work}

In this paper, an improved adaptive backstepping passive coordinated control strategy for generator excitation and STATCOM is designed by adopting I\&I adaptive control, backstepping method, and passivity theory. The uncertainties of parameters and disturbances are taken into account in the coordinated control system. The derivative of the virtual control input is treated as an uncertainty and nonlinear damping algorithm is applied to solve the "calculation explosion" problem inherent in the backstepping method. The proposed coordinated control strategy in this paper only considers the influence of two uncertain parameters on system stability, while the system model also contains other uncertain parameters, such as line reactance, transformer reactance, and electromagnetic power. In the later research, the influences of multiple uncertain parameters in the system will be considered to ensure the stability of the control system. In addition, the situation in practical engineering application is often complicated, and the multimachine power system is usually more likely to work for real. Therefore, this method will be extended in the multimachine power system.

\section{Data Availability}

No data were used to support this study.

\section{Conflicts of Interest}

The authors declare that they have no conflicts of interest.

\section{Acknowledgments}

This work was supported by the National Natural Science Foundation of China (51607133), Shaanxi Science and Technology Plan Project (2020TG-011), and Technology Graduate Innovation Fund Project of Xi'an University (CHX2020019).

\section{References}

[1] X. C. Li and D. Y. Chen, "Nonlinear coordinated control of STATCOM and generator excitation for multi-machine power systems based on improved multiobjective holographic feedback," IEEJ Transactions on Electrical and Electronic Engineering, vol. 13, no. 10, pp. 1-9, 2018.

[2] D. D. Yan, F. F. Wang, J. T. Pan, and W. J. Long, "The voltage control for self-excited induction generator based on STATCOM," Earth and Environmental Science, vol. 153, no. 4, 2018.

[3] R. P. Praveen, J. P. Therattil, J. Jose et al., "Hybrid control of a multi area multi machine power system with facts devices using non-linear modelling," IET Generation Transmission \& Distribution, vol. 14, no. 10, pp. 1993-2003, 2020.
[4] C. Guo, L. Zhong, J. Zhao, and G. Gao, "Single-phase reactive power compensation control for STATCOMs via unknown system dynamics estimation," Mathematical Problems in Engineering, vol. 2020, no. 15, 9 pages, Article ID 8394513, 2020.

[5] O. Ö. Mengi and O. Zdal, "Comparison of MPC based advanced hybrid controllers for STATCOM in medium scale PEM fuel cell systems," International Journal of Hydrogen Energy, vol. 45, no. 43, pp. 23327-23342, 2020.

[6] K. G. Pabitra and B. Abhik, "Stability enhancement of wind energy integrated hybrid system with the help of static synchronous compensator and symbiosis organisms search algorithm," Protection and Control of Modern Power Systems, vol. 5, no. 13, pp. 1076-1083, 2020.

[7] H. Erol, "Delay margin computation of generator excitation control system by using fractional order controller," Transactions of the Institute of Measurement and Control, vol. 42, no. 13, pp. 12465-12474, 2020.

[8] W. L. Li and W. X. Lin, "Nonlinear coordinated control of STATCOM and generator excitation based on improved dynamic surface control method," Applied Mechanics and Materials, vol. 385-386, no. 5, pp. 872-876, 2013.

[9] X. Zhu and J. Wang, "Improving nonlinear coordinated control of non-ideal STATCOM and excitation of salient-pole generator," Research Journal of Applied Sciences, Engineering and Technology, vol. 5, no. 12, pp. 3407-3416, 2013.

[10] L. Cong and Y. Wang, "Coordinated control of generator excitation and statcom for angle stability and voltage regulation enhancement," Iet Proceedings Generation Transmission \& Distribution, vol. 149, no. 6, pp. 3407-3416, 2002.

[11] R. Hemalatha and M. Ramasamy, "Microprocessor and PI controller based three phase CHBMLI based DSTATCOM for THD mitigation using hybrid control techniques," Microprocessors and Microsystems, vol. 76, no. 10, pp. 1638-1648, 2020.

[12] A. Jafari and G. Shahgholian, "Analysis and simulation of a sliding mode controller for mechanical part of a doubly-fed induction generator-based wind turbine," IET Generation, Transmission \& Distribution, vol. 11, no. 10, pp. 2677-2688, 2017.

[13] B. Zou and J. Wang, "Coordinated control for STATCOM and generator excitation based on passivity and backstepping technique," Power System Technology, vol. 35, no. 3, pp. 245-250, 2011.

[14] K. Wang and M. L. Crow, "Hamiltonian theory based coordinated nonlinear control of generator excitation and STATCOM," in North American Power Symposium IEEE, vol. 1, no. 5, Arlington, TX, USA, September 2010.

[15] Q. Su, W. Quan, W. Quan, G. Cai, and J. Li, "Improved robust adaptive backstepping control approach on STATCOM for non-linear power systems," IET Generation, Transmission \& Distribution, vol. 11, no. 13, pp. 3428-3437, 2017.

[16] A. Kanchanaharuthai, "Immersion and Invariance-based non-linear coordinated control for generator excitation and static synchronous compensator of power systems," Electric Power Components and Systems, vol. 42, no. 12, pp. 10041015, 2014.

[17] L.-Y. Sun, S. Tong, and Y. Liu, "Adaptive backstepping sliding mode control of static var compensator," IEEE Transactions on Control Systems Technology, vol. 19, no. 5, pp. 1178-1185, 2011.

[18] R. Ma and G. Zhang, "Sliding mode tracking control with differential evolution optimisation algorithm using integralchain differentiator in uncertain nonlinear systems," 
International Journal of Systems Science, vol. 49, no. 6, pp. 1345-1352, 2018.

[19] T. L. Pan, B. Wu, and Y. S. Chen, "Stability of implicit midpoint algorithm applied to structures with nonlinear damping," Advanced Materials Research, vol. 671-674, no. 5, pp. 1609-1613, 2013.

[20] M. Ali, A. Jalali, and M. G. Naraghi, "Adaptive fuzzy control of a class of nonaffine nonlinear system with input saturation based on passivity theorem," ISA Transactions, vol. 69, no. 12, pp. 202-213, 2017.

[21] M. A. Arjona, R. Escarela-Perez, G. Espinosa-Perez et al., "Validity testing of third-order nonlinear models for synchronous generators," Electric Power Systems Research, vol. 79, no. 9, pp. 953-958, 2009. 